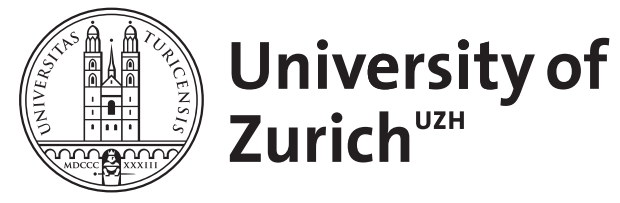

\title{
In vitro Strömungsanalyse von künstlichen Herzklappen
}

Hirt, F ; Botnar, R ; Kepner, J ; Boesiger, $\mathrm{P}$

DOI: https://doi.org/10.1515/bmte.1996.41.s1.54

Posted at the Zurich Open Repository and Archive, University of Zurich ZORA URL: https://doi.org/10.5167/uzh-155073

Journal Article

Published Version

Originally published at:

Hirt, F; Botnar, R; Kepner, J; Boesiger, P (1996). In vitro Strömungsanalyse von künstlichen Herzklappen. Biomedizinische Technik. Biomedical engineering, 41(s1):54-55.

DOI: https://doi.org/10.1515/bmte.1996.41.s1.54 


\title{
In vitro Strömungsanalyse von künstlichen Herzklappen
}

\author{
Hirt F., Botnar R.*, Kepner J.+, Boesiger P.* \\ Strömungstechnik, Sulzer Innotec AG \\ CH-8401 Winterthur, Schweiz \\ *Institut für Biomedizinische Technik und Medizinische Informatik \\ Universität und ETH Zürich, CH-8092 Zürich, Schweiz \\ +CarboMedics Inc., Austin TX, U.S.A.
}

\section{EINLEITUNG}

Künstliche Herzklappen werden auf Grund von physikalischen und numerischen in vitro Experimenten entwickelt und verbessert. Dabei ist es das Ziel, kleine Bewegungen und Deformationen der Fluidpartikel (Blutkörperchen) zu erfassen. Damit können Folgerungen bezüglich Thrombusformation aufgestellt und Prothesen optimiert werden.

In physikalischen Experimenten werden Strömungsgeschwindigkeiten sowie lokale Turbulenzgrössen mit Hilfe von LDA (Laser Doppler Anemometry) oder PIV (Particle Image Velocimetry) gemessen. Eine andere und neuartige Methode, die zur Visualisierung von Blutströmungen entwickelt wurde, ist die MRI (Magnetic Resonance Imaging) Technik. Diese wurde von [1] erstmals zur Messung der stationären Strömung von künstlichen Herzklappen in vitro angewendet. Über die pulsierende Strömung einer Zweiflügel-Aortenklappe ist kürzlich in [2] und [3] berichtet worden. Mit Hilfe eines ausgeklügelten Puls-Duplikator-Systems wurde in [3] die instationäre Strömung nachgebildet und sowohl mit klinischer MRI- als auch mit optischer LDA-Technik untersucht.

\section{SIMULATION UND ANALYSE DES STRÖMUNGSFELDES}

Im physikalischen Experiment wird die physiologische Herztätigkeit des linken Ventrikels und die Ausbreitung der Pulswelle in den Gefässen in einem hydraulischen Kreislauf naturgetreu nachgebildet. Für ein newtonsches Blutanalog-Fluid wird die Physik exakt wiedergegeben. Ein solcher Versuchsaufbau wurde für die Anwendung von optischen Messtechniken (LDA, PIV) entwickelt und in [4] beschrieben. Ein ähnlicher Versuchsaufbau ist für die Anwendung der MRI-Technik angefertigt und in [3] dokumentiert worden. In Figur 1 ist die Strömung einer Zweiflügel-Herzklappe in der Aortenseite dargestellt. Die Bilder sind mit Hilfe eines horizontalen Laser-Lichtschnittes und PolyStyren ${ }^{\text {Ts}}$-Partikel $(60 \mu \mathrm{m})$ erzeugt worden.

Im numerischen Experiment kann die Strömung auch von kleinen Einzelheiten der Klappe untersucht und optimiert werden. Die Auflösung des Strömungsfeldes hängt dabei von der Feinheit des Berechnungsnetzes ab. In Figur 2 ist ein spezielles Berechnungsnetz für eine Zweiflügel-Herzklappe dargestellt. Dieses Netz wurde für die transiente Strömungssimulation mit zwangsgesteuerten Flügeln entwickelt. Da zur Lösung der Grund- gleichungen physikalische und numerische Näherungen erforderlich sind, muss die numerische Simulation immer mit Hilfe von physikalischen Experimenten validiert werden. Mit steigender Komplexität der nachzubildenden Natur (Pulsation, Turbulenz, Fluid-ZellenInteraktion, Biochemie) werden deshalb sorgfältig ausgelegte in vitro und in vivo Experimente benötigt.
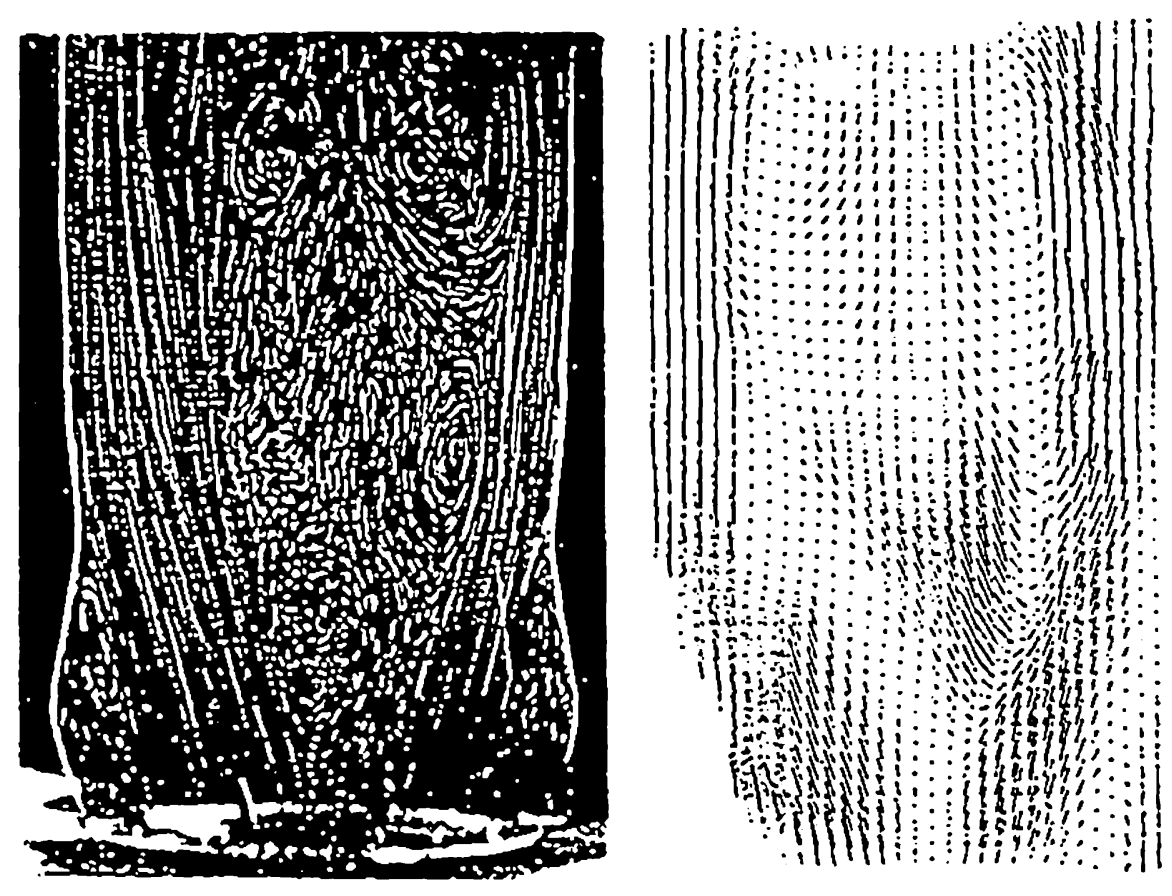

Fig.1 Ebener Schnitt durch das momentane Strömungsfeld einer mechanischen Zweiflügel-Herzklappe. Links: Teilchenbahnen. Rechts: Stromlinien, die mit Hilfe von PIV-Technik in [4] erzeugt worden sind.

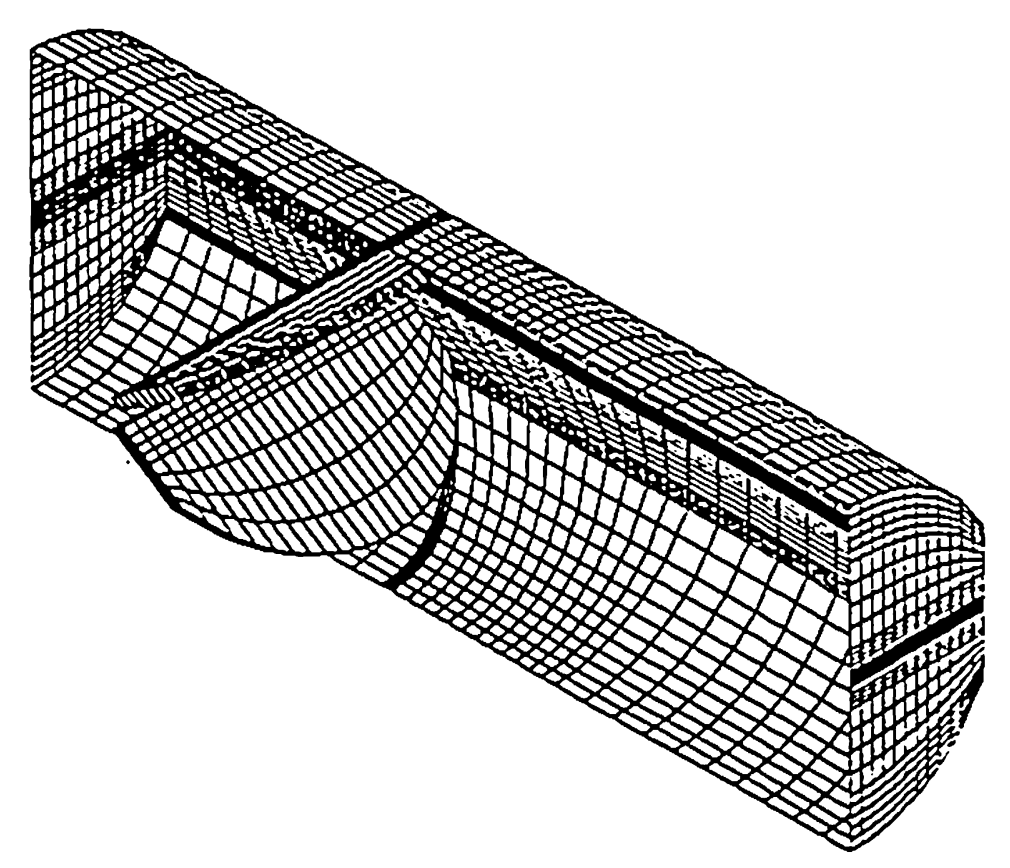

Fig.2 Aufbau des Berechnungsnetzes zur transienten Simulation des 3D-Strömungsfeldes einer ZweiflügelHerzklappe mit bewegten Flügeln [5]. 
